\title{
Application of Imaging Guidelines in Patients With Foreign Body Ingestion or Inhalation: Literature Review
}

\author{
Roberto Grassi, MD, * Angela Faggian, MD, " Francesco Somma, MD,* \\ Carlo Nicola De Cecco, MD, ${ }^{\dagger}$ Andrea Laghi, MD, ${ }^{\dagger}$ and Filipe Caseiro-Alves, $\mathrm{MD}^{\ddagger}$
}

\begin{abstract}
Ingestion, inhalation, and insertion of foreign bodies (FBs) are very common clinical occurrences. In any case, early diagnosis and prompt management are mandatory to avoid severe and life-threatening complications. Radiologists have an important role in revealing the presence, dimension, nature, and relationship with anatomical structures of a FB; selecting the most appropriate imaging modality; and enabling the best therapeutic choice. This review article focuses on the most frequent FBs ingested, inhaled, and inserted and presents the different tests and investigations to provide a correct radiological approach.

Semin Ultrasound CT MRI 36:48-56 @ 2014 Elsevier Inc. All rights reserved.
\end{abstract}

\section{Introduction}

— oreign bodies (FBs) are uncommon radiological findings

- but remain an important reason for admittance to emergency department.

FBs may be inhaled, ingested, or inserted into a body cavity, or they may be deposited into the body by a traumatic or iatrogenic injury.

Inhalation of FBs is frequent among infants and preschool children. It has been estimated that approximately 500 children die each year in the United States owing to inhalation of FBs. ${ }^{1}$ Complications associated with a delayed diagnosis are pneumonia, atelectasis, bronchiectasis, and bronchial fistula. ${ }^{2,3}$

Ingestion is the most common occurrence, and it has been estimated that up to 1500 deaths occur annually in the United States owing to ingestion of FBs. Most of these cases occur in pediatric population before the age of 6 years and in adults between 25 and 44 years. ${ }^{4}$

In most cases, FBs cause only mild mucosa injury. It has been reported that $80 \%-90 \%$ of FBs that reach gastrointestinal

*Institute of Radiology, Second University of Naples, Naples, Italy.

$†$ Department of Radiological Sciences, Oncology and Pathology, University of Rome Sapienza, Latina, Italy.

\#Universitary Clinic of Radiology, Coimbra University Hospitals, Coimbra, Portugal.

Address reprint requests to Angela Faggian, MD, Institute of Radiology, Second University of Naples, Piazza Miraglia 2, Naples 80138, Italy. E-mail: angela. faggian@libero.it
(GI) tract will pass through it without difficulty, 10\%-20\% require nonoperative intervention, and less than 1\% need surgery. $^{5-7}$

Complications such as perforation, bowel obstruction, fistula, bleeding, abscess formation, and peritonitis may occur, depending on the type and size of the object. Even if they are uncommon, the associated morbidity may be severe and lifethreatening. ${ }^{4}$

Insertion of $\mathrm{FB}$ is no longer a medical oddity; it is encountered frequently and is associated with a significant morbidity and mortality. ${ }^{8,9}$

To prevent complications, early diagnosis based on a correct radiological documentation and interpretation of inhalation, ingestion, and insertion of FBs is mandatory.

In this article, we discuss the most common FBs in terms of epidemiology, pathogenesis, clinical aspects, and radiological findings. In addition, we suggest the imaging modality of choice to improve management of patients, decreasing related complications.

\section{Inhaled FBs}

Inhalation of FBs in children is a potentially serious household accident that constitutes a frequent complaint especially in pediatric emergency rooms. ${ }^{10}$

In Italy, in the year 2005, according to data available from the Ministry of Health, 356 patients had diagnosis of "FBs in 
Table 1 National Data of Ministry of Health in the 2005 About Patients Discharged With Diagnosis of FB in Trachea, Bronchi, and Lungs

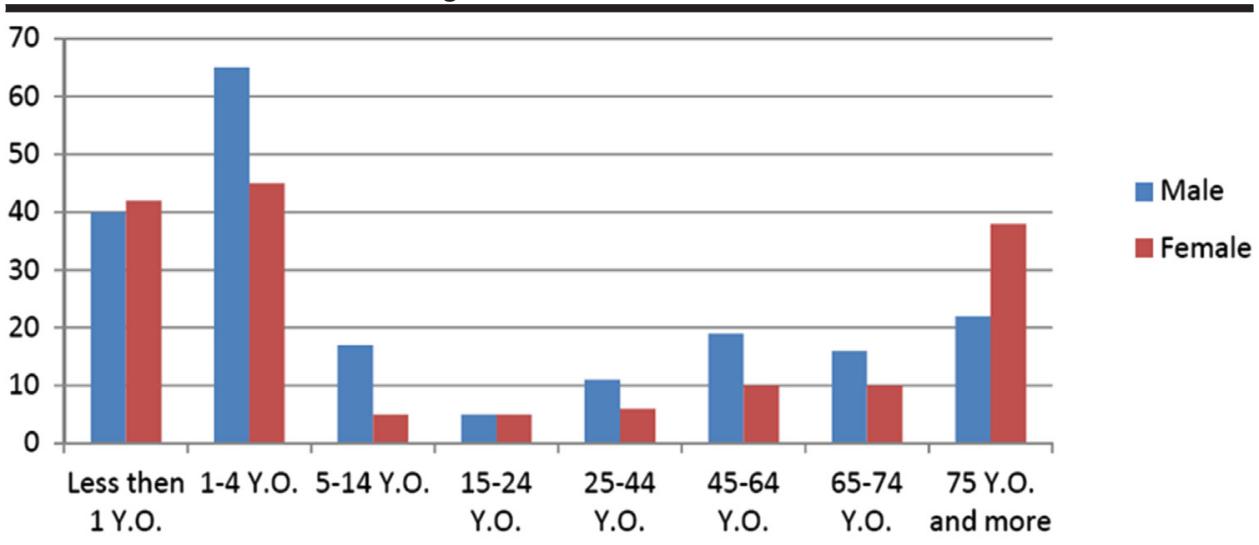

Y.O.: years old

trachea, bronchi, and lungs," with a significant prevalence (53\%) in children younger than 4 years (Table 1 ).

Many authors ${ }^{11,12}$ report that more than $80 \%$ of cases of inhalation of FBs occur during early childhood, with a peak incidence between 10 and 24 months of age.

In addition, official US statistics from 1995 refer to mechanical choking caused by FBs in the airway as being responsible for $5 \%$ of deaths among children younger than 4 years. The absence of molars and premolars and the tendency to put all objects in the mouth account for the particular predisposition of children of this age group. ${ }^{13}$

On the contrary, inhalation of FBs is rare in adults, in whom it is often associated with specific pathologic conditions such as mental retardation, seizures, Parkinson disease, and brain tumors and also to therapeutic dental procedures performed with patients under local anesthesia and in the supine position. ${ }^{14}$ In addition, FB inhalation could be a rare presentation of myasthenia gravis. Weakness of laryngeal and pharyngeal musculature leads to ineffective swallowing with poor airway protection, and this may be further compounded by a poor cough because of respiratory musculature involvement. The combination of poor respiratory effort and an ineffective swallowing with no protective mechanisms can lead to choking and eventually inhalation. ${ }^{15}$

\section{Clinical Features}

The most frequent symptom is the "penetration syndrome," defined as a sudden onset of choking and intractable cough with or without vomiting. ${ }^{16}$ The clinical presentation has a wide spectrum from no symptoms in the case of small objects being deposited deep down in the airways to respiratory failure when the objects are located at the epiglottis or the carina. Hence, the location of the FB is important in symptom development. When the location is the supra-glottis, sign and symptoms are cough, dyspnea, salivation, voice changes; when in the larynx, there are stridor, cough, voice changes, and severe difficulty breathing; when in the intrathoracic trachea, physical examination reveals expiratory wheeze and inspiratory rhonchus; when in the extrathoracic trachea, inspiratory stridor and expiratory rhonchus are observed; and when in the bronchus, cough, wheezing or other localized sounds, and difficulty breathing are noted. ${ }^{17}$ Inhalation, which does not have any acute clinical presentation, can remain undetected and occult for a long time. These patients are misdiagnosed and treated with antibiotics and bronchodilators several times, as inhalation of FBs can mimic many diseases such as croup, asthma, pneumonia, bronchitis, or lung cancer. Delayed diagnosis, common in case of occult inhaled FBs, ranges from hours to years, leading to chronic complications as bronchiectasis, bronchoesophageal fistula, persistent pneumonia, or abscess. Thus, complicated patients may undergo surgical pulmonary procedures. ${ }^{18}$ For these reasons, patients with inhaled FBs require rapid diagnosis and prompt treatment.

\section{Diagnostic Workflow}

When FB inhalation is suspected, chest $\mathrm{x}$-ray is often performed as the first choice. Metallic objects are readily identified on chest radiographs. However, most inhaled FBs are nonradiopaque. A radiolucent FB can be suggested only by indirect findings, such as segmental or lobar collapse, air trapping in a unilateral hyperlucent lung, postobstructive lobar or segmental infiltrates, or other chronic pulmonary changes. ${ }^{19}$ An expiratory chest x-ray should be requested when the standard inspired film result is normal, as this strategy allows visualization of air trapped by a valvelike effect due to partial obstruction of the bronchial lumen. ${ }^{17,20}$ Airway fluoroscopy, allowing respiratory movements, has limited value in this situation. Chest x-ray for FB inhalation has a sensitivity that ranges from $60 \%-85 \%$ and a specificity from $52 \%-68 \% .^{21,22}$ However, these findings are nonspecific as they may occur even in patients without FB inhalation. Negative chest x-ray result in patients with a strong clinical suspicion of FB inhalation requires bronchoscopy. ${ }^{23}$ Bronchoscopy is the most specific and sensitive diagnostic procedure and may also provide the ultimate treatment. However, there are several complications associated with this technique that require general anesthesia in pediatric patients. ${ }^{24}$ Rigid bronchoscopy complications range between $2 \%$ and $22 \%$ and are frequently 


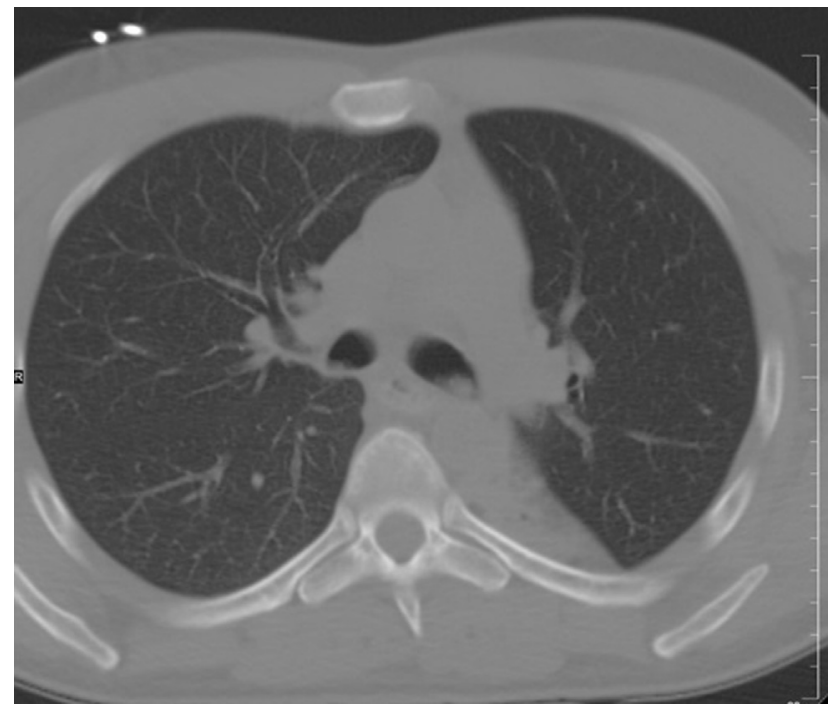

Figure 1 Axial CT image below the carina reveals foreign body in the left main bronchus and ipsilateral pleural effusion.

represented by laryngeal edema and pneumothorax. More serious complications such as tracheal or bronchial tear, hypoxia, and cardiorespiratory arrest can also occur. Luckily, these complications remain exceptional, particularly for experienced pediatric bronchoscopy teams. ${ }^{25}$ The diagnostic algorithm comprising flexible bronchoscopy has reduced the negative rigid bronchoscopy finding rate to $2 \%{ }^{26}$

Multidetector computed tomography (MDCT) with virtual bronchoscopy is a noninvasive diagnostic technique with sensitivity and specificity of almost $100 \%$, as it is able to show the lumen of the tracheobronchial tree and detect radiolucent FBs with accuracy similar to rigid bronchoscopy, identifying the exact location of the FB impaction ${ }^{21,27}$ (Fig. 1). Furthermore, it can correctly detect parenchymal findings (Fig. 2) and allow an optimal visualization of the airways distal to the obstruction. $^{21,24}$

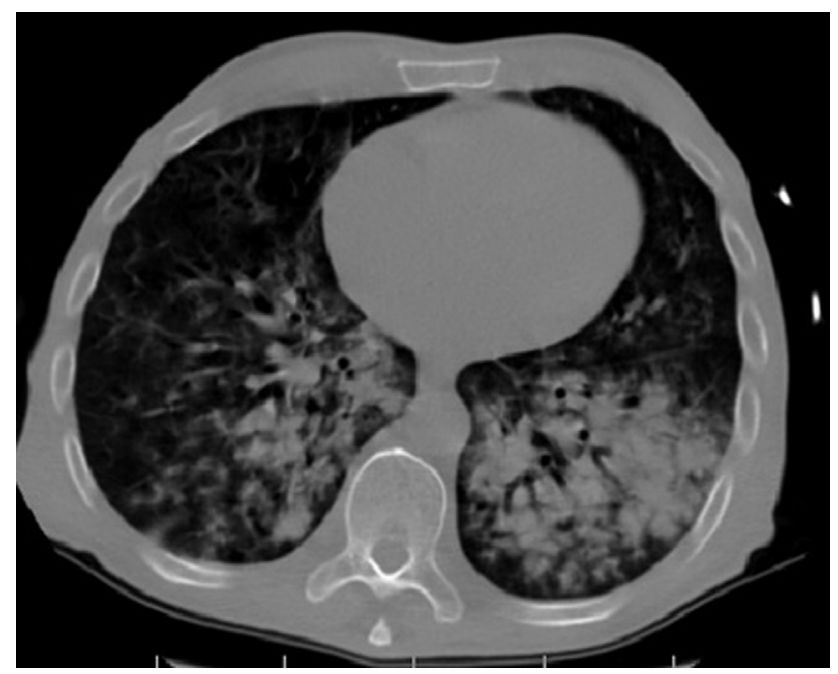

Figure 2 CT scan of lower lobe bronchi: multiple nodular opacities bilaterally and areas of "tree in bud."
Moreover, several authors have reported that MDCT may be burdened by false-positive rates, for calcified lymph nodes misinterpreted as endobronchial FBs, ${ }^{19}$ and that it therefore should not be routinely used as the initial diagnostic approach. $^{23}$ Differential diagnosis includes tracheobronchial obstruction due to external airway compression for the presence of enlarged lymph nodes, mediastinal neoplasms, or cardiomegaly or due to intraluminal tracheobronchial obstruction for the presence of bronchiolitis, neoplasm, granulation tissue as in tuberculosis, mucus plugs and secretions as in bacterial bronchopneumonia, cystic fibrosis, asthma, pulmonary abscess, or acute laryngotracheobronchitis. ${ }^{28}$

If chest $\mathrm{x}$-ray proves a FB inhalation, there is no need to perform MDCT examination.

The indication for MDCT is still valid in the case of clinical suspicion of FB inhalation, in patients with negative chest radiography finding, or in the case of atypical presentation and discrepancy between clinical and radiological findings. ${ }^{23}$ If MDCT examination reveals no FB in the tracheobronchial tree, bronchoscopy may be avoided ${ }^{21}$ (Table 2).

Magnetic resonance imaging (MRI) has a unique indication in the suspicion of peanuts inhalation, as its high fat-rich content has a high signal on T1-weighted images in comparison with surrounding air in the lung, which has a low signal, allowing high contrast between the FB and the air in the lung.

Furthermore, MRI allows to distinguish between peanuts and indirect findings such as granulation or local atelectasis, which usually appear as isointense on Tl-weighted image scans. On the contrary, it is difficult to distinguish them using MDCT, because they appear nearly identical in brightness. ${ }^{29,30}$

\section{Ingested FBs}

Ingestion of FB is a common clinical problem. In Italy, in the year 2005, 1666 patients had diagnosis of "FB in the digestive tract" (Table 3).

Most of the cases occur in the pediatric population, with a peak incidence between 6 months and 4 years in relation to the oral stage of learning, inadequate dentition, the relatively high position of epiglottis, and immature swallowing coordination. ${ }^{31,32}$ A second peak occurs in adults aged 25-44 years (9.5\%) with a prevalence in men. At this age, it can be an accidental event mainly in persons with decreased palatal sensitivity, owing to the use of false teeth, excessive alcohol intake, and extremely cold fluid intake; those with swallowing disorders; or those with psychiatric disorders and drug addiction. Intentional episodes occur in mentally handicapped people, drug traffickers, and prisoners. ${ }^{33}$

A correlation has been found between age groups and specific types of ingested objects. Coins, toys, crayons, and ballpoint pen caps are most often ingested during the childhood, whereas adults tend to have problem with meat and bones. Moreover, psychiatric patients and prison inmates may ingest such unlikely objects as spoons and razor blades. ${ }^{34}$ In addition, oral ingestion of drugs is a widespread and growing practice of illegally transporting cocaine and other narcotics. 
Table 2 Suggested Management of Patient With FB Inhalation

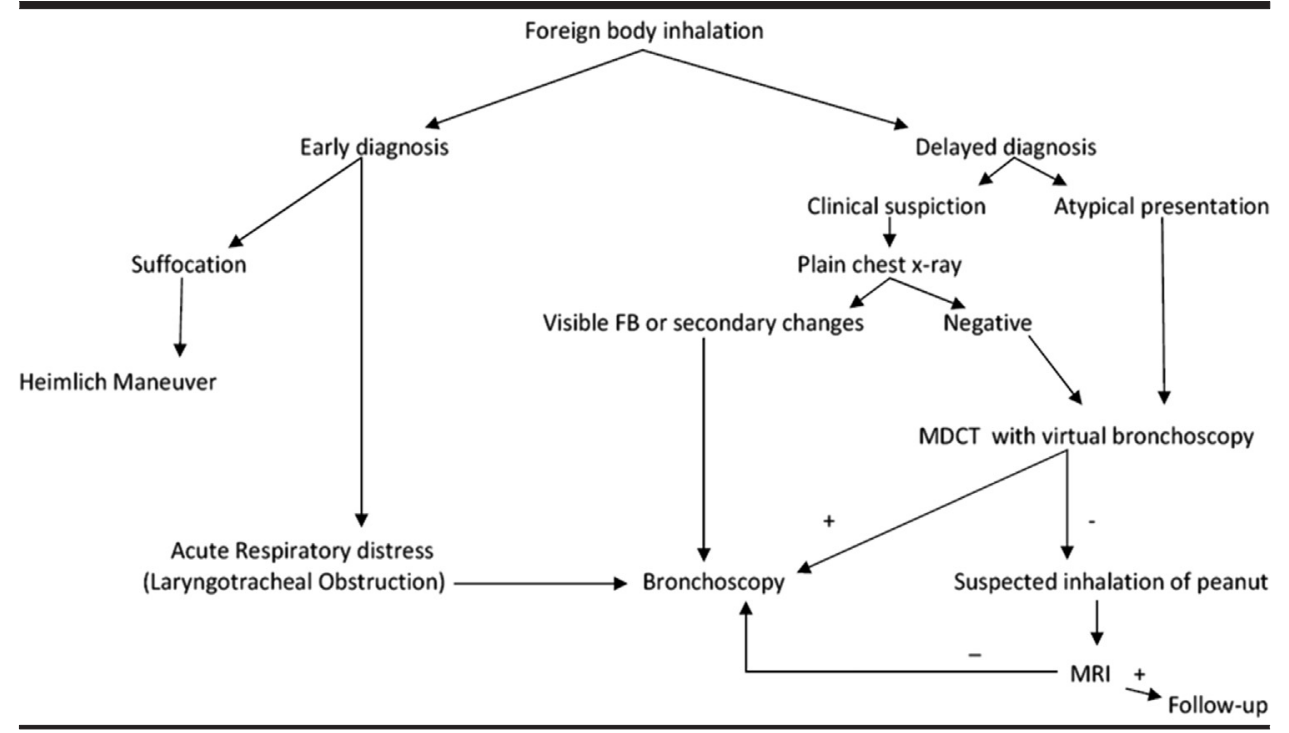

People who engage in this practice for purpose of transport across borders and subsequent retrieval of the drug in a foreign country are commonly known as body packers or mules. Differently from a body packer, a body stuffer is someone who hastily ingests illegal drugs to escape detection by authorities. ${ }^{35-37}$ Drug traffickers can swallow between 40 and 150 packets of illegal drugs, with total cash values ranging from $\$ 50,000$ to more than $\$ 1,000,000$. $^{35}$

If an ingested FB successfully navigates the esophagus, it will frequently pass through the entire GI tract. Moreover, if the object has irregular or sharp edges, it may lodge anywhere in the GI tract, causing mechanical obstruction frequently at areas of anatomical narrowing, physiological angulation, or areas of pathologic structures. If the objects are elongated, they usually become trapped in the duodenum, appendix, or ileocecal valve. Spherical or cylindrical objects tend to be large and impact in the esophagus or pylorus. Perforation is another possible complication, and the most common sites are the lower esophagus, duodenal loop, appendix, ileocecal valve, and the recto-sigmoid region. Fistula, abscess, peritonitis, or generalized septicemia is usually a result of perforation. ${ }^{38,39}$ The reported incidence of FBs causing perforation of the GI tract is less than $1 \%$, with the objects being elongated or sharp in most of the cases, such as toothpicks, pins, fish, or chicken bones. Furthermore, with long, narrow, and pointed ingested FBs, the risk of perforation could be higher; this occurrence may not only be the result of direct mechanical erosion, as with bones, but also of chemical corrosion, as with button batteries. In addition, patients with previous GI tract surgery or congenital gut malformations are at increased risk. ${ }^{40,41}$

Table 3 National Data of Ministry of Health in the 2005 About Patients Discharged With Diagnosis of FB in the Digestive Tract

\section{NATIONAL DATA IN THE YEAR 2005 \\ ICDM 938:FB in the digestive tract}

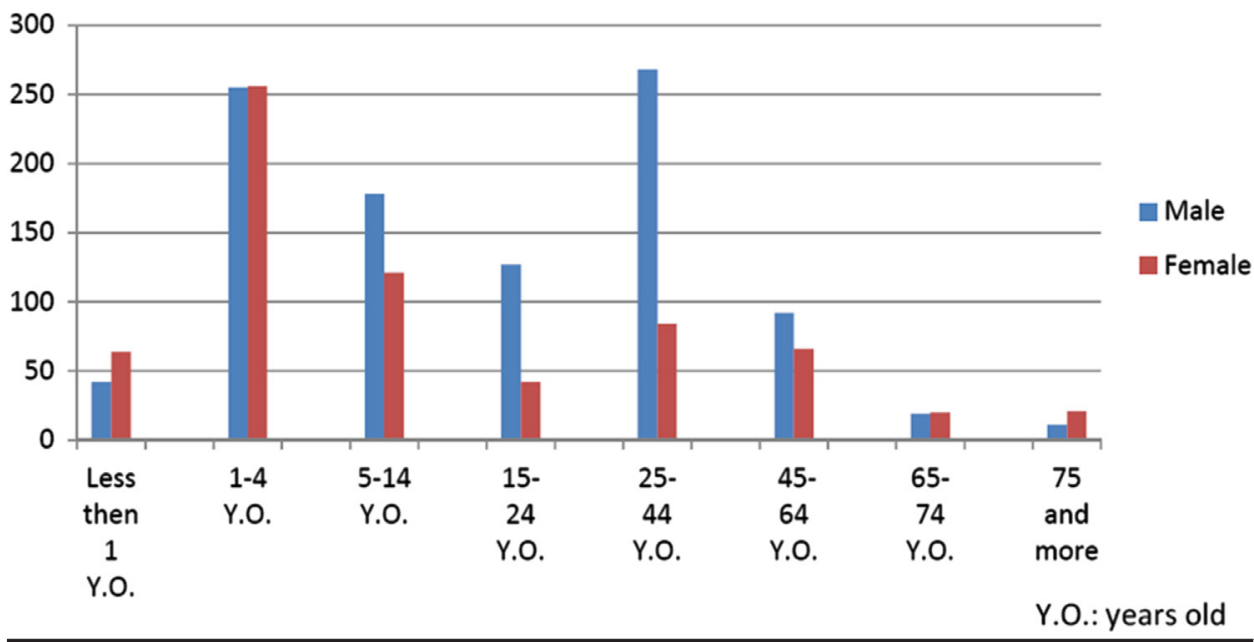




\section{Clinical Features}

Clinical presentation is influenced by anatomical location; type, size, duration of impaction of ingested FBs; and related complications, such as obstruction, perforation, and lethal drug exposure.

Approximately $80 \%$ of the cases of obstruction caused by ingested FBs occur in the hypopharynx or the cervical esophagus, causing discomfort and pain in cervical region. ${ }^{42}$ Objects that lodge in the esophagus can cause airway obstruction, stricture, or perforation with resulting mediastinitis, cardiac tamponade, paraesophageal abscess, or aortotracheoesophageal fistula. ${ }^{34}$ An understanding of the risk factors for complication associated with esophageal FBs might help to reduce patient morbidity. A multivariate analysis showed that the duration of impaction and type and size of FBs $(P<0.001)$ were significant independent risk factors associated with the development of complications in patients with esophageal FB. ${ }^{4}$ Morbidity and mortality associated with body packing can occur owing to bowel obstruction and intoxication following packet rupture. For this reason, it is required to adopt a systematic protocol for the management of these patients (Table 4). ${ }^{43}$

When the perforation of GI tract occurs, it may give peritonitis, abscess, inflammatory mass formation, obstruction, fistulae, and hemorrhage. ${ }^{44}$ In addition, it could even involve adjacent structures such as the kidneys, psoas muscles, and inferior cava. ${ }^{45}$ Rare cases of FB migration to the pleura, heart, kidney, or liver have been reported. ${ }^{46-50}$

Lethal drug exposure may also occur in body stuffers, even if large quantities of cocaine are not involved, owing to the poor wrapping of drugs with condoms, latex, cellophane, and other synthetic materials. ${ }^{37}$

\section{Diagnostic Workflow}

Many patients who have swallowed FBs are without knowledge when symptoms occur, and physicians must maintain a high index of suspicion. Radiologists have an important role in revealing the presence, dimension, nature, and relationship with anatomical structures of a FB; selecting the most appropriate imaging modality; and enabling the best therapeutic choice.

Radiographs of antero-posterior and lateral chest, lateral neck, and supine abdominal should be obtained to rule out ingestion. In total, 2 projections are mandatory because some FBs, especially those of discoid shape, could be shown only in one projection, remaining undetectable in the other one.

Table 4 Management of Ingestion of Suspected Drug Packets

Suspected body packer

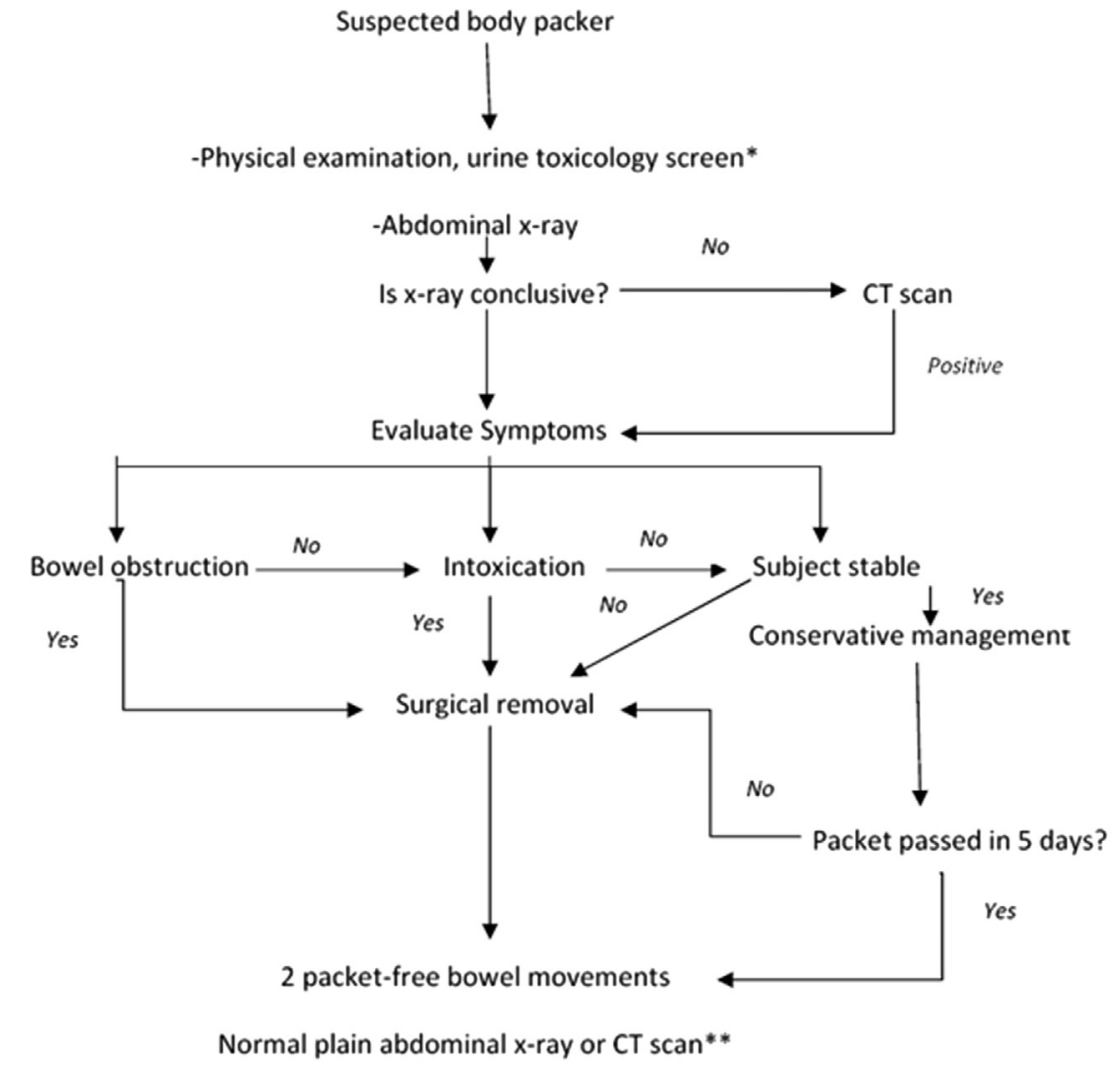

*urine toxicology screening may be positive because the body packer is often also a drug user 


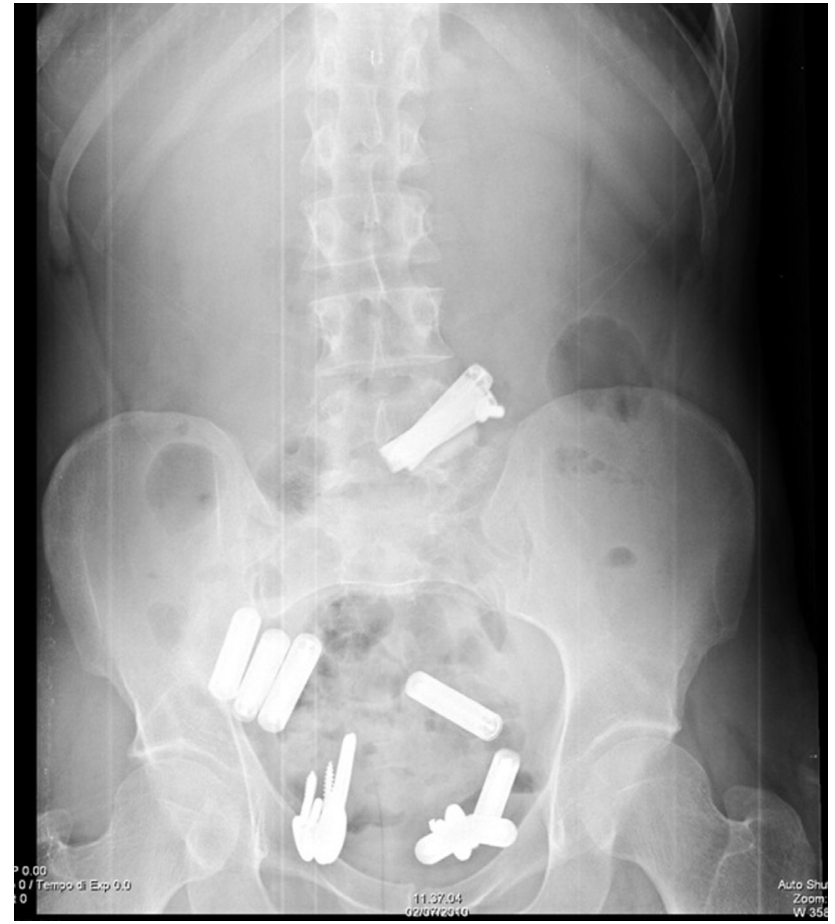

Figure 3 Plain abdominal x-ray: numerous radiopaque images with metallic density in the presumable ileal loops (batteries and keys).

Radiological visualization depends on radiopacity. ${ }^{38,51}$ Radiograph detects as much as $80 \%$ of all FBs. Objects of metal, except aluminum, most animal bones, and glass are opaque on radiographs (Figs. 3-6). Objects composed of

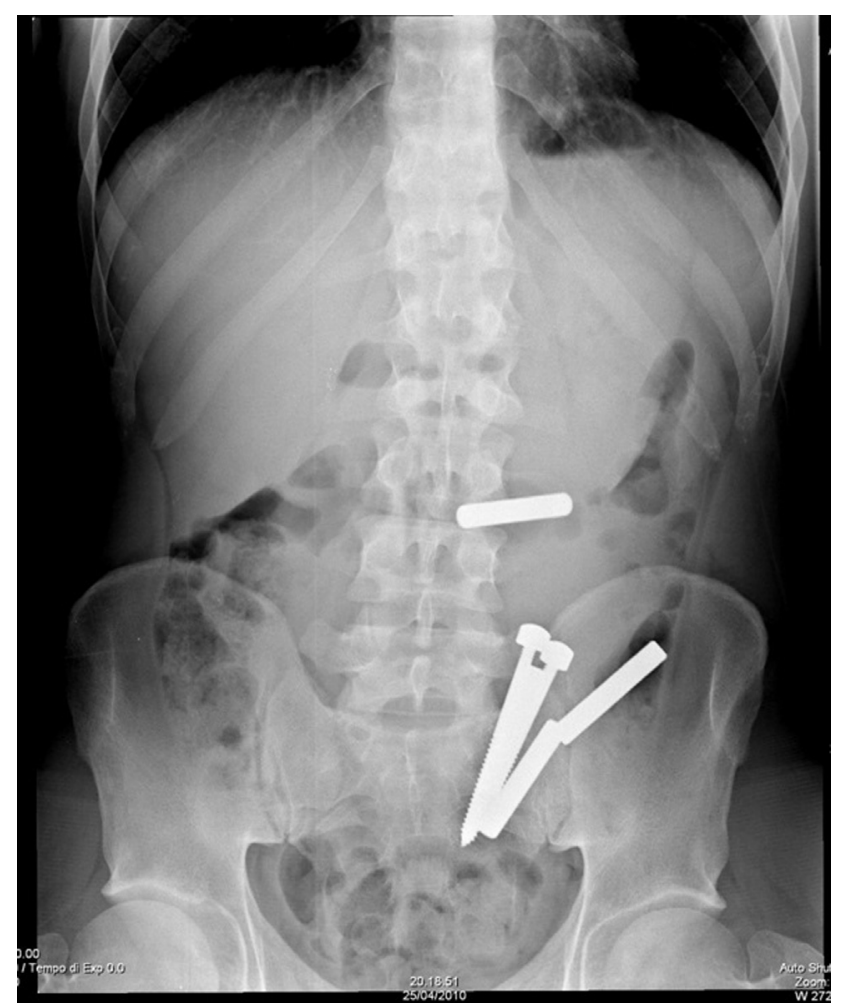

Figure 4 Plain abdominal x-ray: radiopaque images (3 screws + 2 hinges) in the small bowel loops.

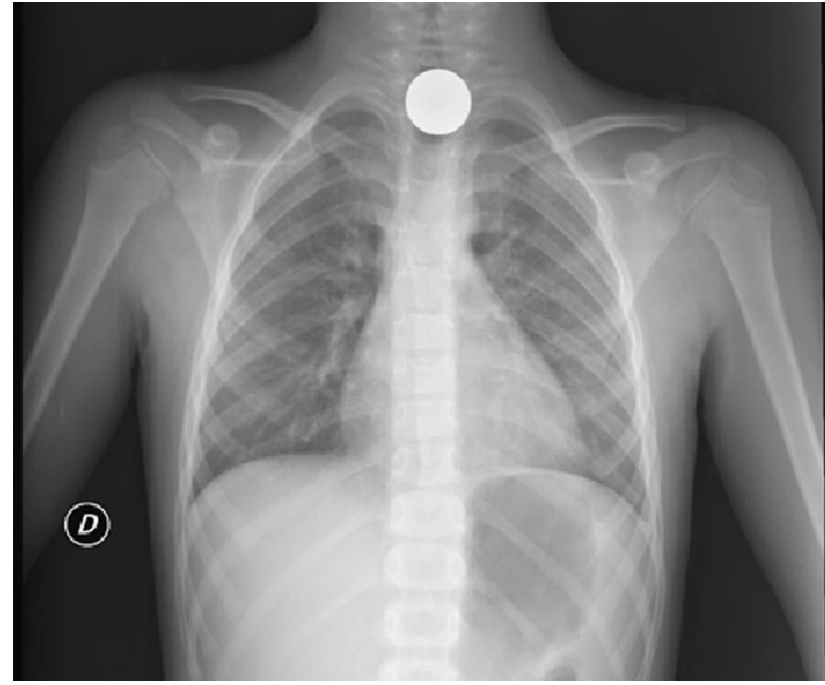

Figure 5 Chest x-ray: radiopaque foreign body (coin) at the junction between the esophagus and hypopharynx.

plastic and most fish bones are radiolucent structures, and their diagnosis may be challenging. ${ }^{3}$

Aluminum objects may be radiographically detected with sensitivity and specificity from $80 \%-90 \%$ and from 90\%$100 \%$, respectively, and could be easily missed on a superficial reading of the images. ${ }^{52}$

Regarding FBs as fish bone, chicken bone, and toothpicks, $\mathrm{x}$-ray has a sensitivity that ranges from $23.5 \%-54.8 \%$ for the first 2 and $9 \%$ for the latter. In case of toothpicks, even other imaging studies have a low sensitivity, 15\% for MDCT and $29 \%$ for ultrasound (US), which are the methods of choice in

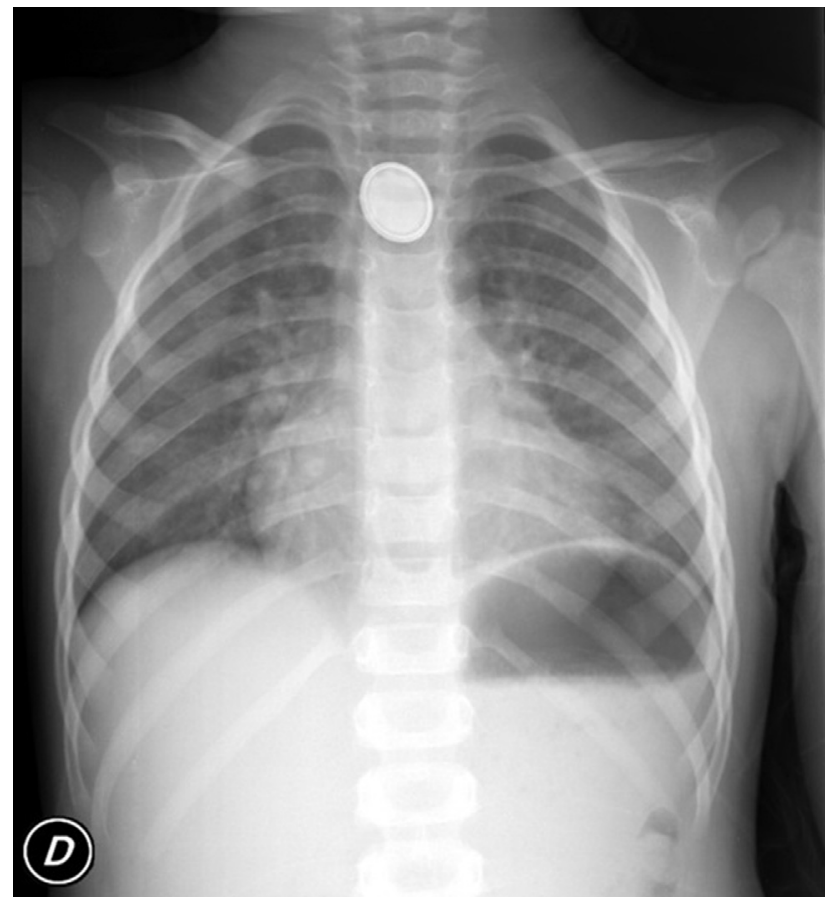

Figure 6 Chest x-ray: radiopaque foreign body in the lower third of the esophagus. It is probably a button battery, demonstrating the "halo sign" of a smaller disc within a larger disc. 
the diagnosis of a FB that migrated from the GI tract and retained in the soft tissues. ${ }^{41}$

If an oral contrast medium is administered, sensitivity increases. In this case, water-soluble contrast medium is preferable to barium for the high risk of perforation. In the absence of clear signs of extraluminal extravasation of the water-soluble contrast medium, additional radiographs can be performed after the oral administration of barium. Experimental and clinical studies have shown that small pharyngoesophageal perforations by ingested FBs can be very accurately visualized using barium. ${ }^{53}$ Nevertheless, a false-positive rate of $27 \%-50 \%$ and false-negative rate of $19 \%-40 \%$ have been reported.$^{54}$ In addition, the use of barium is associated with a high risk of inhalation, and along with coating of FB and esophageal mucosa, it could compromise subsequent endoscopy. ${ }^{41}$ Therefore, MDCT examination is mandatory in the management of this condition, in identifying high-density FBs. Moreover, MDCT not only confirms the existence and the location of any impacted fish bones, it also enables the visualization of any resulting damage to neighboring structures.

MDCT should also be performed in all patients with suspected fish bone impaction in the GI, even in cases in which plain x-ray has already established the presence of a FB. MDCT may provide useful information not only for the diagnosis of ingested FBs but also for the detection of complications. The region of perforation may appear as a thickened focal segment, associated with localized pneumoperitoneum, regional fatty infiltration, and associated intestinal obstruction. Furthermore, it represents the best strategy for the management of these patients. In fact, surgical removal of FBs from the esophagus is required within 8 hours, otherwise it can result in exacerbation of a perforation of the esophageal wall. This kind of complication can be avoided by performing MDCT to determine whether a FB is still in the esophageal lumen or has penetrated in its wall. ${ }^{41,55}$ However, the accuracy of MDCT is limited by the lack of observer awareness, and a high index of suspicion should always be maintained for a correct diagnosis to avoid interpretation mistakes. Further limitations of MDCT are given by slice scanning thickness: the use of thinner MDCT slices allows reviewers to better trace structure such as blood vessels and distinguish them from calcified FBs. The orientation of a FB with respect to an axial MDCT scan can also affect the perception of the viewer. Coronal reconstruction would be useful especially in overcoming this limitation. ${ }^{56}$

MRI is suggested only in preoperative assessment in patients with soft tissue masses, providing excellent soft tissue contrast and an optimal visualization of anatomical extent of the mass and its relation to adjacent structures, even without the administration of intravenous contrast medium. ${ }^{57}$

US, abdominal x-ray, MDCT, and MRI are used to examine people suspected of being body packers; however, the sensitivity and specificity of these examinations are yet unknown. Examining the bowel for small FBs with US is problematic as the drug-filled packets may have a similar appearance to stool and air and are characterized as an echogenic structure with a shadowing. ${ }^{43}$ Abdominal $x$-ray is the most used examination to detect drug-filled packets. Radiological density depends on the

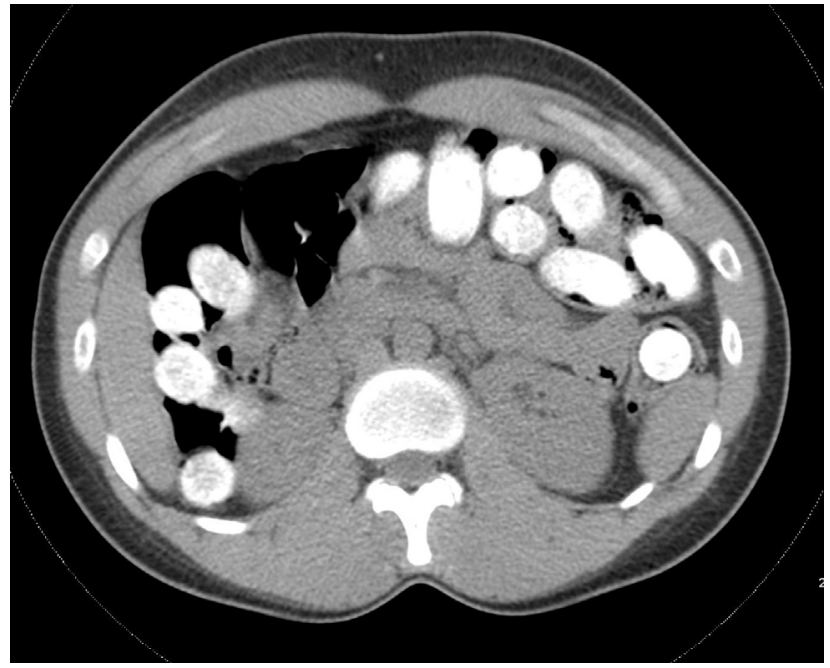

Figure 7 Abdominal CT scan in patient with several drug-filled packets

composition with other substances or on its grade of purity. This is the reason why abdominal $\mathrm{x}$-ray is occasionally unable to identify the packets. Improvements in packaging and increasing sophistication of traffickers also make radiological visualization of the packets more difficult. ${ }^{58}$

Therefore, MDCT has been reported to be more accurate than radiography in the detection of the drug-filled packets because of its improved contrast resolution and the elimination of projections of overlapping structures (Fig. 7). Nevertheless, false-negative MDCT results are also described. In fact, identifying the homogeneous isodense heroin packets is really a difficult problem. They may go undetected if the window width and level settings typically employed for abdominal MDCT (ie, window width $400 \mathrm{HU}$ and window level $40 \mathrm{HU}$ ) are used. For this reason, in cases of ingestion of suspected homogeneous isodense drug packet, it is advisable to manipulate the windows (level $-150 \sim-300 /$ width $600 \sim 800$ ) in addition to those commonly used for abdominal MDCT. Moreover, the detection of changes in size and shape of the lumen is helpful as indirect signs..$^{5,60}$

\section{Conclusion}

Inhalation, ingestion, or insertion of FBs are very common clinical problems.

To prevent complications, early diagnosis is mandatory.

Imaging methods are of paramount importance in identifying and locating the FBs, allowing the selection of the best therapeutic option, namely conservative, endoscopic, laparoscopic, or open surgical methodologies.

It is important for any radiologist to be aware of the typical imaging findings of FBs to select the most appropriate imaging modality for their detection, depending on their nature.

\section{References}

1. Li Y, Wu W, Yang X, et al: Treatment of 38 cases of foreign body aspiration in children causing life-threatening complications. Int J Pediatr Otorhinolaryngol 73(12):1624-1629, 2009 
2. Reilly J, Thompson J, MacArthur C, et al: Pediatric aerodigestive foreign body injuries are complications related to timeliness of diagnosis. Laryngoscope 107(1):17-20, 1997

3. Yadav SPV, Singh J, Aggarwal N, et al: Airway foreign body in children: Experience of 132 cases. Singapore Med J 48(9):850-853, 2007

4. Sung SH, Jeon SW, Kim HK, et al: Factor predictive of risk for complications in patients with esophageal foreign bodies. Dig Liver Dis 43(8):632-635, 2011

5. Aoyagi K, Maeda K, Morita I, et al: Endoscopic removal of a spoon from the stomach with a double-snare and balloon. Gastrointest Endosc 57(7): 990-991, 2003

6. Blaho KE, Merigian KS, Winbery SL, et al: Foreign body ingestions in the emergency department: Case reports and review of treatment. J Emerg Med 16(1):21-26, 1998

7. Velitchkov NG, Grigorov GI, Losanoff JE, et al: Ingested foreign bodies of the gastrointestinal tract: Retrospective analysis of 542 cases. World J Surg 20(8):1001-1005, 1996

8. Huang WC, Jiang JK, Wang HS, et al: Retained rectal foreign bodies. J Chin Med Assoc 66(10):607-612, 2003

9. Busch DB, Starling JR: Rectal foreign bodies: Case reports and a comprehensive review of the world's literature. Surgery 100(3): 512-519, 1986

10. Sadan N, Raz A, Wolach B: Impact of community educational programmes on foreign body aspiration in Israel. Eur J Pediatr 154(10): 859-862, 1995

11. Kadmon G, Stern Y, Bron-Harlev E, et al: Computerized scoring system for the diagnosis of foreign body aspiration in children. Ann Otol Rhinol Laryngol 117(11):839-843, 2008

12. Zhijun C, Fugao Z, Niankai Z, et al: Therapeutic experience from 1428 patients with pediatric tracheobronchial foreign body. J Pediatr Surg 43(4):718-721, 2008

13. Rovin JD, Rodgres BM: Pediatric foreign body aspiration. Pediatr Rev 21(3):86-90, 2000

14. Yilmaz A, Akkaya E, Damadoglu E, et al: Occult bronchial foreign body aspiration in adults: Analysis of four cases. Respirology 9(4):561-563, 2004

15. Patel U, Forsen J: Foreign body aspiration: An unusual presentation of myasthenia gravis. Otolaryngol Head Neck Surg 124(6):698-699, 2001

16. Baharloo F, Veyckemans F, Francis C, et al: Tracheobronchial foreign bodies: Presentation and management in children and adults. Chest 115(5):1357-1362, 1999

17. Lima JAB, Fisher GB: Foreign body aspiration in children. Paediatr Respir Rev 3(4):303-307, 2002

18. Ulku R, Baskan Z, Yavuz I: Open surgical approach for a tooth aspirated during dental extraction: A case report. Aust Dent J 50(1): 49-50, 2005

19. Zissin R, Shapiro-Feinberg M, Rozenman J, et al: CT findings of the chest in adults with aspirated foreign bodies. Eur Radiol 11(4):602-611, 2001

20. Girardi G, Contador AM, Castro-Rodriguez JA: Two new radiological findings to improve the diagnosis of bronchial foreign-body aspiration in children. Pediatr Pulmonol 38(3):261-264, 2004

21. Haliloglu M, Ciftci AO, Oto A, et al: CT virtual bronchoscopy in the evaluation of children with suspected foreign body aspiration. Eur J Radiol 48(2):188-192, 2003

22. Koşucu P, Ahmetoğlu A, Koramaz I, et al: Low-dose MDCT and virtual bronchoscopy in pediatric patients with foreign body aspiration. Am J Roentgenol 183(6):1771-1777, 2004

23. Pinto A, Scaglione M, Pinto F, et al: Tracheobronchial aspiration of foreign bodies: Current indications for emergency plain chest radiography. Radiol Med 111(4):497-506, 2006

24. Jung SY, Pae SY, Chung SM, et al: Three-dimensional CT with virtual bronchoscopy: A useful modality for bronchial foreign bodies in pediatric patients. Eur Arch Otorhinolaryngol 269(1):223-228, 2012

25. Ciftci AO, Bingöl-Koloğlu M, Senocak ME, et al: Broncoscopy for evaluation of foreign body aspiration in children. J Pediatr Surg 38 (8):1170-1176, 2003

26. Martinot A, Deschildre A, Brichet A, et al: Indications of bronchial endoscopy in suspected tracheo-bronchial foreign body in children. Rev Mal Respir 16(4 Pt 2):673-678, 1999
27. Hong SJ, Goo HW, Roh JL: Utility of spiral and cine CT scans in pediatric patients suspected of aspirating radiolucent foreign bodies. Otolaryngol Head Neck Surg 138(5):576-580, 2008

28. Tokar B, Ozkan R, Ilhan H: Tracheobronchial foreign bodies in children: Importance of accurate history and plain chest radiography in delayed presentation. Clin Radiol 59(7):609-615, 2004

29. Imaizumi H, Kaneko M, Nara S, et al: Definitive diagnosis and location of peanuts in the airways using magnetic resonance imaging techniques. Ann Emerg Med 23(6):1379-1382, 1994

30. O'Uchi T, Tokumaru A, Mikami I, et al: Value of MR imaging in detecting a peanut causing bronchial obstruction. Am J Roentgenol 159(3): 481-482, 1992

31. Laguna D, González FM: Calcification of the posterior cricoid lamina simulating a foreign body in the aerodigestive tract (2005:11b). Eur Radiol 16(2):515-517, 2006

32. Heyer CM, Bollmeier ME, Rossler L, et al: Evaluation of clinical, radiologic, and laboratory prebronchoscopy findings in children with suspected foreign body aspiration. J Pediatr Surg 41(11):1882-1888, 2006

33. Pinto A, Muzj C, Stavolo C, et al: Pictorial essay: Foreign body of the gastrointestinal tract in emergency radiology. Radiol Med 107(3): $145-154,2004$

34. Orting M, Lundbech PE, Bjerregaard NC, et al: Presentation, diagnosis and management of swallowed foreign bodies. Ugeskr Laeger 176(2): $157-162,2014$

35. Traub SJ, Hoffman RS, Nelson LS: Body packing-the internal concealment of illicit drugs. N Engl J Med 349(26):2519-2526, 2003

36. Beckley I, Ansari NA, Khwaja HA, et al: Clinical management of cocaine body packers: The Hillingdon experience. Can J Surg 52(5):417-421, 2009

37. Fineschi V, Centini F, Monciotti F, et al: The cocaine "body stuffer" syndrome: A fatal case. Forensic Sci Int 126(1):7-10, 2002

38. Bartalena T, Rinaldi MF, Rinaldi G: Ingested aluminium foreign body. Eur J Radiol Extra 71(1):23-24, 2009

39. Reddy SK, Griffith GS, Goldstein JA, et al: Toothpick impaction with localized sigmoid perforation: Successful colonoscopic management. Gastrointest Endosc 50(5):708-709, 1999

40. Benjamin SB: Small bowel obstruction and the Garren-Edwards gastric bubble: An iatrogenic bezoar. Gastrointest Endosc 34(6):463-467, 1988

41. Webb W: Management of foreign bodies of the upper gastrointestinal tract: Update. Gastrointest Endosc 41(1):39-51, 1995

42. Pinto A, Muzj C, Gagliardi N, et al: Role of imaging in the assessment of impacted foreign bodies in the hypopharynx and cervical esophagus. Semin Ultrasound CT MR 33(5):463-470, 2012

43. Mandava N, Chang RS, Wang JH, et al: Establishment of a definitive protocol for the diagnosis and management of body packers (drug mules). Emerg Med J 28(2):98-101, 2011

44. Romano L, Palumbo P, Rossi G, et al: An unusual case of a liver abscess cured by radiological endoscopic procedures. Radiol Med 87(6):893-895, 1994

45. Nigri GR, Di Giulio E, Di Nardo R, et al: Duodenal perforation and right hydronephrosis due to toothpick ingestion. J Emerg Med 34(1):55-57, 2008

46. Santos SA, Alberto SC, Cruz E, et al: Hepatic abscess induced by foreign body: Case report and literature review. World J Gastroenterol 13(9): 1466-1470, 2007

47. Theodoropoulou A, Roussomoustakaki M, Michalodimitrakis MN, et al: Fatal hepatic abscess caused by a fish bone. Lancet 359(9310):977, 2002

48. Meyns BP, Faveere BC, Van de Werf FJ, et al: Constrictive pericarditis due to ingestion of a toothpick. Ann Thorac Surg 57(2):489-490, 1994

49. Shaffer RD: Subcutaneous emphysema of the leg secondary to toothpick ingestion. Arch Surg 99(4):542-545, 1969

50. Ma J, Kang DK, Bae JI, et al: Value of MDCT in diagnosis and management of esophageal sharp or pointed foreign bodies according to level of esophagus. Am J Roentgenol 201(5):W707-W711, 2013

51. ASGE Standards of Practice Committee, Ikenberry SO, Jue TL, et al: Management of ingested foreign bodies and food impaction. Gastrointest Endosc 73(6):1085-1091, 2011

52. Valente JH, Lemke $\mathrm{T}$, Ridlen M, et al: Aluminium foreign bodies: Do they show up on x-ray? Emerg Radiol 12(1-2):30-33, 2005 
53. Foley MJ, Ghahremani GG, Rogers LF: Reappraisal of contrast media used to detect upper gastrointestinal perforations. Radiology 144(2):231-237, 1982

54. Derowe A, Ophir D: Negative findings of esophagoscopy for suspected foreign bodies. Am J Otolaryngol 15(1):41-45, 1994

55. Akazawa Y, Watanabe S, Nobukiyo S, et al: The management of possible fishbone ingestion. Auris Nasus Larynx 31(4):413-416, 2004

56. Goh BK, Tan YM, Lin SE, et al: CT in the preoperative diagnosis of fish bone perforation of the gastrointestinal tract. Am J Roentgenol 187(3): 710-714, 2006
57. Scaglione M, Pinto F, Grassi R, et al: Migration of a foreign body from the pharynx to the soft tissues of the neck: Delayed presentation with Homer's syndrome. Am J Roentgenol 172(4):1131-1132, 1999

58. Hergan K, Kofler K, Oser W: Drug smuggling by body packing: What radiologists should know about it. Eur Radiol 14(4):736-742, 2004

59. Yang RM, Li L, Feng J, et al: Heroin body packing: Clearly discerning drug packets using CT. South Med J 102(5):470-475, 2009

60. Pinto A, Reginelli A, Pinto F, et al: Radiological and practical aspects of body packing. Br J Radiol 87(1036):20130500, 2014 